\title{
Preferências e tabus alimentares no consumo de pescado em Santarém, Brasil
}

\section{Feeding preferences and taboos related to the fish consumption at Santarém, Brazil}

Tony Marcos Porto Braga - Doutor em Ecologia pelo Instituto Nacional de Pesquisas da Amazônia/INPA. Professor no Instituto de Ciências e Tecnologia das Águas da Universidade Federal do Oeste do Pará /UFOPA, Brasil. E-mail: tony.braga@gmail.com

Adrielly Alves da Silva - Graduanda em Engenharia de Pesca no Instituto de Ciências e Tecnologia das Águas pela Universidade Federal do Oeste do Pará/UFOPA, Brasil. E-mail: ellyalves92@hotmail.com

George Henrique Rebêlo - Doutor em Ecologia pela Universidade Estadual de Campinas. Pós-doutorado pela Universidade de Wageningen. Pesquisador titular e coordenador do Laboratório de Manejo de Fauna do Instituto Nacional de Pesquisas da AmazôniaINPA, Brasil. E-mail: jacarebelo@gmail.com

\section{Resumo}

Foi registrado o uso de recursos pesqueiros pelos moradores da cidade de Santarém-PA, no baixo rio Amazonas, e analisados os aspectos associados à evitação ou ao incremento de seu consumo. Os dados foram coletados mensalmente nos mercados e feiras, mediante a aplicação de questionários semiestruturados junto a consumidores e comerciantes de pescado, entre agosto de 2013 e julho de 2014. Os dados foram armazenados em um banco de dados relacionais e submetidos à estatística descritiva. $\mathrm{O}$ peixe foi o principal produto adquirido e comercializado $(80 \%$ dos entrevistados). Atualmente, preferem o tambaqui e o tucunaré - e não o acari e a pescada, que eram os preferidos antigamente. $\mathrm{O}$ tabu tradicional contra o consumo de peixes lisos ainda existe, mas algumas espécies que antes não eram consumidas ou pouco consumidas estão sendo aceitas e encontradas nos mercados.

\section{Palavras-chave}

Recursos pesqueiros. Preferências alimentares. Amazônia. Tabus.

\begin{abstract}
The use of fishing resources by the residents of the city of Santarém-PA in the lower Amazon was recorded and were analyzed the avoidance or the increase of consumption. Data were collected monthly in the markets and fairs, through the application of semistructured questionnaires to consumers and fish merchants between August 2013 and July 2014. The data were stored in a relational database and submitted to descriptive statistics. The fish was the main product purchased and sold ( $80 \%$ of respondents). Currently tambaqui and the peackok tucunare are the prefered fish and not the acari and the freshwater hake that were formerly preferred. The traditional taboo against the consumption of catfish still exists, but some species that were not consumed or little consumed are currently being accepted and available on the market.
\end{abstract}

\section{Keywords}

Fishing resources. Food preferences. Amazon. Taboos. 


\section{INTRODUÇÃO}

O entendimento sobre como os seres humanos procuram, obtêm e escolhem o alimento é necessário para compreender valores culturais e relações sociais (BEGOSSI; PETRERE Jr., 1988). As pessoas reconhecem e classificam os alimentos, e essa classificação influencia os sistemas tradicionais de subsistência, definindo o que deve ser considerado alimento que, por sua vez, é usualmente classificado em uma escala de preferências, que pode determinar tanto a intensidade quanto a frequência com que as espécies são caçadas, pescadas ou cultivadas e consumidas (COSTA-NETO, 2000; SILVANO; BEGOSSI, 2001).

As escolhas alimentares podem ser influenciadas por preferências individuais, fatores ecológicos, econômicos, sociais e culturais, bem como por aversões (MACBETH; LAWRY, 1997). A pesca para obtenção do alimento na Amazônia ocorre em dois sistemas diferentes, considerando a sua sazonalidade e a ecologia das espécies alvo: a pesca nos lagos de várzea e a pesca nos canais dos rios (ISAAC; BARTHEM, 1995; BARTHEM; GUERRA; VALDERRAMA, 1995). Durante a enchente, a água dos lagos transborda e os pescadores permanecem nas áreas de florestas alagadas para onde a maioria das espécies de peixes se desloca em busca de alimento, refúgio e proteção de predadores (BRAGA; REBÊLO, 2014). Quando as águas começam a baixar, os peixes vão procurar abrigo nos corpos de água remanescentes e realizam migrações dispersivas, principalmente Characiformes e Siluriformes, sendo esta uma época que os pescadores capturam mais peixes e obtêm mais renda (RIBEIRO; PETRERE, 1990; ISAAC; BARTHEM, 1995; BATISTA et al., 2004).

Na região amazônica o peixe é a principal fonte de proteína das populações ribeirinhas e o consumo de pescado estimado em 500g/dia (BATISTA et al., 1998; FABRÉ; ALONSO, 1998; BRAGA; BARROS; CHAVES, 2008), que é um dos maiores valores de consumo de pescado no mundo e indicador da forte relação dos populações ribeirinhas com os recursos pesqueiros (BATISTA et al., 2004). Porém, na Amazônia há restrições, não esclarecidas, ao consumo de algumas espécies de peixes. Certos peixes não são consumidos em determinados períodos, por serem considerados "reimosos" (SMITH, 1979). A reima pode ser caracterizada como um sistema classificatório amplo de oposições binárias entre alimentos perigosos (reimosos) e não perigosos (não reimosos) para pessoas em estado físico ou social de liminaridade, assim chamados os estados de representação ritual e simbólica de transição ou passagem, como enfermidades, menstruação e pós-parto (MURRIETA, 2001). 
Esse sistema de classificação depende de crença, pois apesar de não haver evidências científicas indicando que o consumo de certas espécies cause problemas de saúde, a aversão ao consumo de certos peixes ainda é comum na Amazônia, como, por exemplo, os chamados peixes-lisos, principalmente Pimelodidae, Siluriformes. Talvez por este motivo o consumo desses peixes tem sido mínimo na região, e a pesca artesanal do peixe-liso nunca visou abastecer o mercado regional, mas sim vender a produção fresca para os frigoríficos situados desde o Baixo Amazonas até o Alto Solimões (GOULDING, 1979; SMITH, 1979; BITTENCOURT; COX-FERNANDES, 1990; RUFFINO; ISAAC, 1994; RUFFINO; BARTHEM, 1996; BARTHEM, 1997; FABRÉ; ALONSO, 1998).

Até o final da década de 1970 não havia exemplares pequenos de peixes nos mercados, praticamente só exemplares adultos eram comercializados, como o caso dos tambaquis, que eram comercializados geralmente acima do tamanho mínimo de $55 \mathrm{~cm}$ (PETRERE, 1983). Para os pescadores, eles não precisavam pescar exemplares juvenis porque havia peixes adultos para capturar. Espécies de baixo valor comercial eram evitadas ou consumidas pelos próprios pescadores, que faziam a seleção prévia pela escolha do método e da técnica de captura (BAYLEY, 1981; BATISTA; FREITAS, 2003).

Hoje é cada vez mais comum encontrar peixes de diferentes tamanhos - e de espécies que antes eram evitadas - à venda nas feiras e mercados de Santarém. Se as mudanças no comportamento dos pescadores na exploração dos estoques pesqueiros têm influência direta na dinâmica da populacional das espécies (BATISTA et al., 2004), então esta situação merece ser pesquisada e avaliada. Este estudo teve o objetivo de registrar o uso atual de recursos pesqueiros pelos moradores da cidade de Santarém, no baixo Amazonas, e analisar os aspectos associados à evitação ou ao incremento do consumo de certas espécies.

\section{METODOLOGIA}

Este estudo foi realizado nos mercados e feiras da cidade de Santarém, no Pará (S2²4'52"/W5442'36") (Figura 1). Este município possui uma população aproximada de 294.580 habitantes, dos quais 215.790 estão na área urbana (IBGE, 2010). O passo inicial foi consultar a Secretaria Municipal de Agricultura e Abastecimento (SEMAB) para identificar os mercados e feiras nas diferentes zonas existentes da cidade onde havia comercialização de pescado. Os dados foram coletados semanalmente nos mercados e feiras (Mercadão 2000, Mercado Modelo e Feira do Pescado ou "tablado"), em dias alternados, durante um ano, entre agosto de 2013 e julho de 2014, mediante a aplicação 
de questionários semiestruturados junto aos consumidores e comerciantes de pescado. Foram aplicados 228 questionários, sendo 115 para comerciantes e 113 para consumidores, sendo entrevistadas, de preferência, pessoas com idade acima de 40 anos.

As informações coletadas foram: espécies preferidas para o consumo, espécies não consumidas ou pouco consumidas, motivos da escolha, outros usos para os peixes que não o consumo, historicidade da quantidade das espécies (se aumentou ou diminuiu, na visão dos entrevistados). Os dados coletados foram armazenados em banco de dados relacionais elaborado na plataforma Access, sendo em seguida submetidos à estatística descritiva, para o cálculo da média, desvio padrão $( \pm)$ e frequência de ocorrência (\%) (GONZÁLES et al., 2006).

A análise qualitativa dos dados foi realizada com base na categorização do conteúdo das respostas, segundo o modelo de associação das diversas competências individuais (MARQUES, 1991), o qual consiste em considerar todas as informações fornecidas por todos os entrevistados, sem exclusão. A preferência pelas espécies de peixes antes e atualmente consumidas foi comparada através da razão de verossimilhança (Teste-G) (GONZÁLES et al., 2006).

Figura 1. Localização do município de Santarém, Pará.

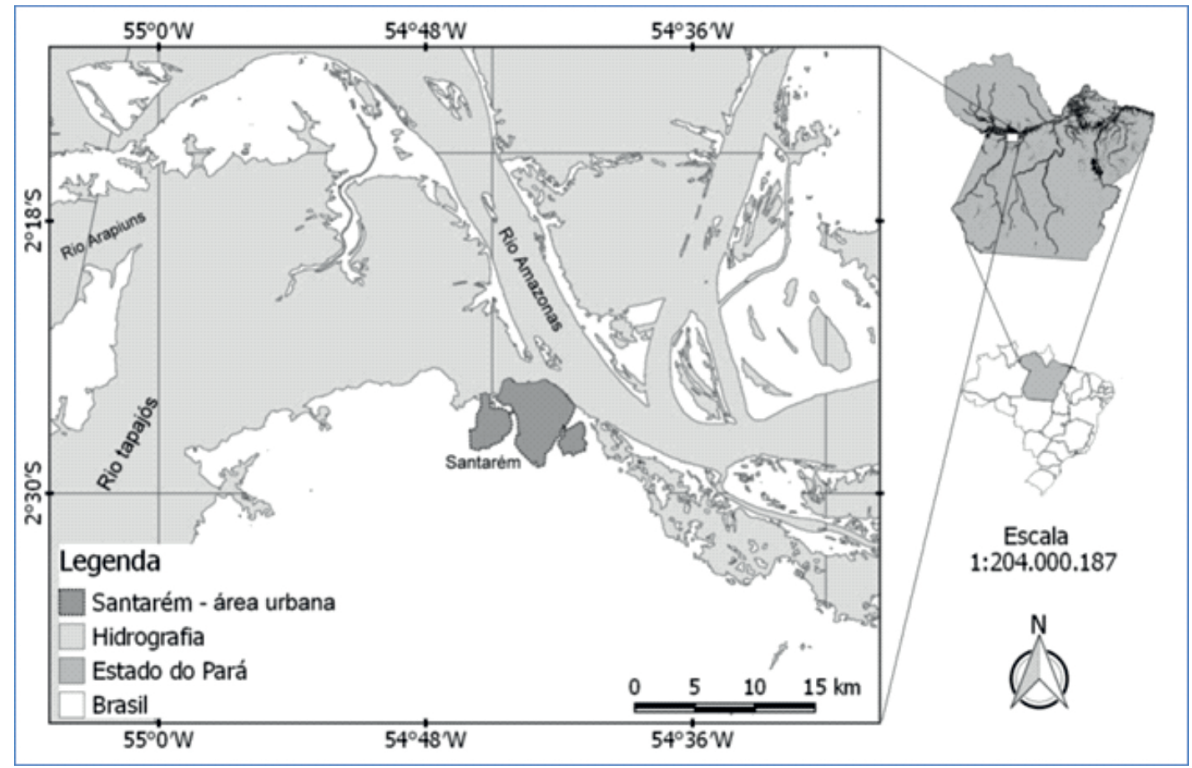

Fonte: Laboratório de Geoinformação e Análise Ambiental (ICTA/UFOPA). 


\section{RESULTADOS E DISCUSSÃO}

A idade média dos consumidores entrevistados foi de 57,08 ( $\pm 10,97)$ anos e dos comerciantes $52( \pm 12,90)$. O peixe foi o principal produto adquirido por $80 \%$ dos consumidores entrevistados, que foram aos mercados em busca de pescado a ser utilizado apenas como alimento. Os demais entrevistados adquiriram, além do peixe, outros produtos como piracuí (farinha de peixe) e camarão (Figura 2). Os entrevistados que adquiriram camarões os diferenciavam em dois tipos - o camarão comum (Macrobrachium amazonicum) e o pequeno avium (Acetes americanus).

Figura 2. Pescado adquirido pelos consumidores em mercados e feiras de Santarém.

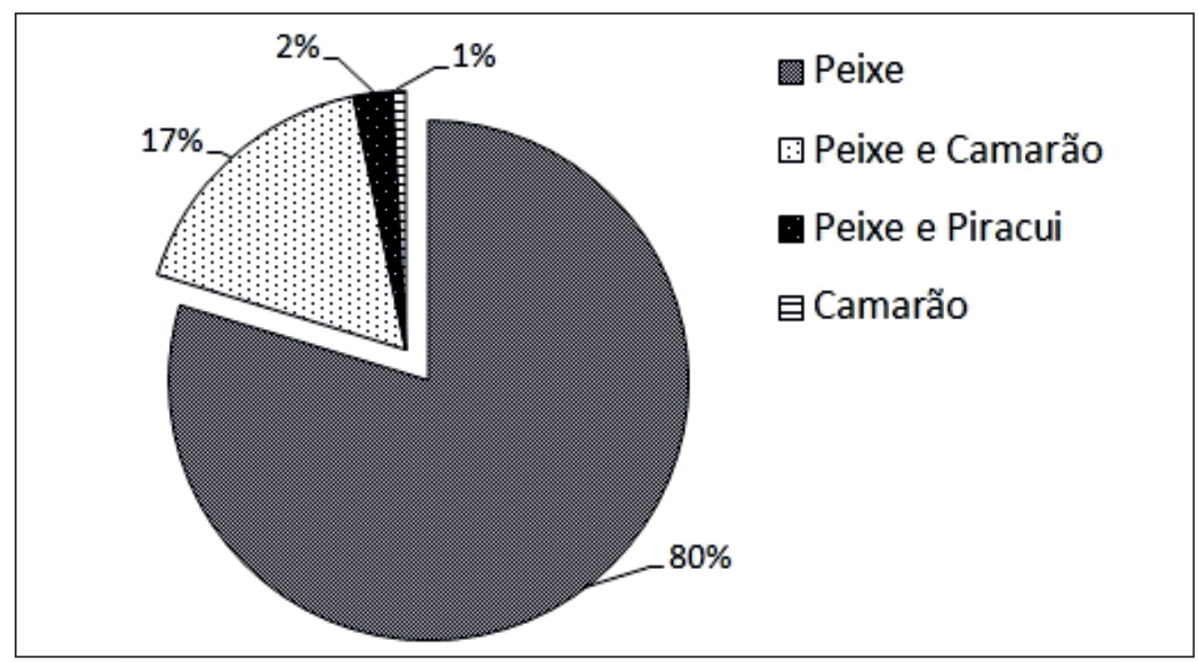

Fonte: Laboratório de Geoinformação e Análise Ambiental (ICTA/UFOPA).

Entre os 38 tipos de peixes citados para o consumo (Tabela 1), destacaramse os denominados "peixes de escama", como o tambaqui (Colossoma macropomum), tucunaré (Cichla spp.), curimatã (Prochilodus nigricans) e pacu (Mylossoma spp., Metynnis sp., Myleus sp.) como sendo os preferidos (Figura 3). Os comerciantes citaram justamente essas três primeiras espécies mais vendidas, como sendo as preferidas pelos consumidores e para o seu próprio consumo. Os motivos alegados para essas preferências estão relacionados principalmente ao sabor (80,5\%), por serem espécies mais “saudáveis", e por não serem consideradas “reimosas" e, portanto, não prejudiciais à saúde. 
Figura 3. Dez tipos de peixes citados como preferidos para o consumo atualmente em Santarém.

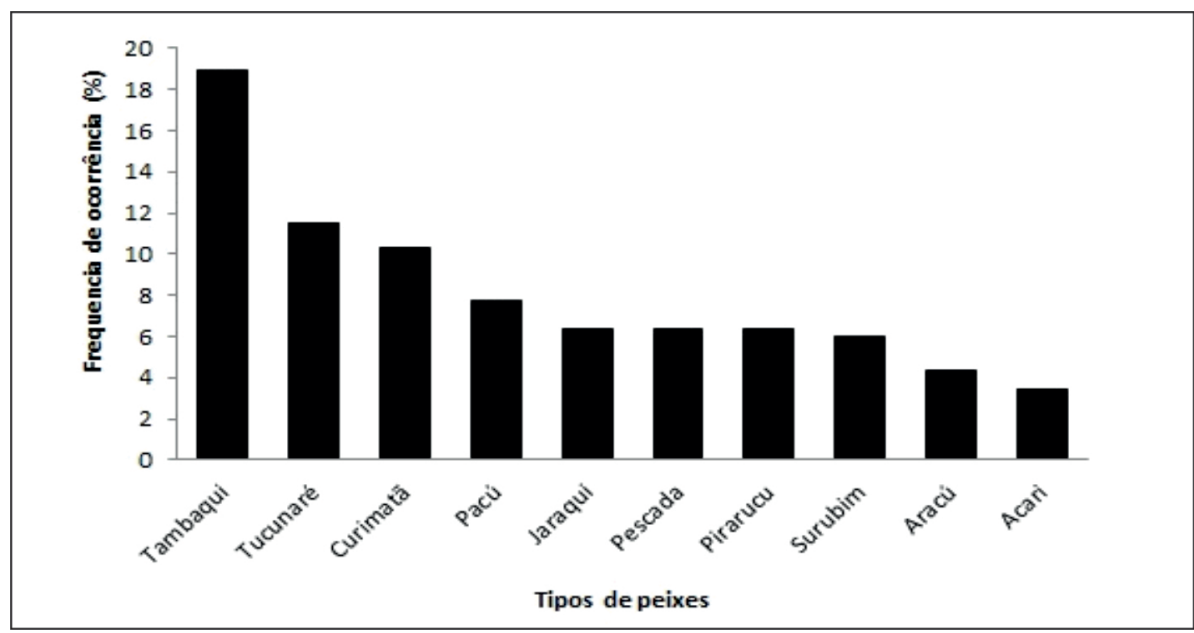

Fonte: Laboratório de Geoinformação e Análise Ambiental (ICTA/UFOPA).

Tabela 1 - Lista de peixes citados para o consumo atualmente em Santarém.

\begin{tabular}{l|l|l}
\hline Ordem / Família & Nome Científico & Nome local \\
\hline Osteoglossiformes & & \\
\hline Osteoglossidae & Osteoglossum bicirrhosum & Aruanã \\
\hline Arapaimatidae & Arapaima gigas & Pirarucu \\
\hline Clupeiformes & & \\
\hline Pristigasteridae & Pellona spp & Apapá ou sarda \\
\hline Characiformes & & \\
\hline Anostomidae & Leporinus spp.; Schizodon spp. & Aracu \\
\hline Characidae & Brycon melanopterus & Jatuarana \\
\hline & Brycon amazonicus & Matrinchã \\
\hline & Mylossoma spp.; Myleus spp. & Pacu \\
\hline & Piaractus brachypomus & Pirapitinga \\
\hline Prochilodontidae & Colossoma macropomum & Tambaqui \\
\hline Hemiodontidae & Prochilodus nigricans & Curimata \\
\hline Siluriformes & Hemiodos spp. & Jaraqui \\
\hline Doradidae & & Cubiu ou charuto \\
\hline & & \\
\hline Loricariidae & Oxydoras niger & \\
\hline & Pterygoplichthys spp. & Cujuba \\
\hline & Rineloricaria spp. & Bodó \\
\hline & & Acari-cachimbo \\
\hline
\end{tabular}




\begin{tabular}{l|l|l}
\hline Pimelodidae & Pseudoplatystoma tigrinum & Caparari \\
\hline & Pseudoplatystoma punctifer & Surubim \\
\hline & Pimelodina flavipinnis & Fura-calça \\
\hline & Hypophthalmus spp. & Mapará \\
\hline & Brachyplatystoma filamentosum & Filhote ou piraíba \\
\hline Auchenipteridae & Brachyplatystoma rousseauxii & Dourada \\
\hline Callichthyidae & Parauchenipterus galeatus & Cangati \\
\hline Perciformes & Ageneiosus spp & Mandubé \\
\hline Cichlidae & Hoplosternum litoralle & Tamoatá \\
\hline & & \\
\hline & Astronotus spp. & Acará-açu \\
\hline & Vários Gêneros & Cará \\
\hline Rajiformes & Cichla spp. & Tucunaré \\
\hline Potamotrygonidae & Plagioscion squamosissimus & Pescada \\
\hline & & \\
\hline & Potamotrygon spp & Arraia \\
\hline
\end{tabular}

Duas categorias gerais identificadas compõem o sistema de classificação local de peixes: peixes de escama e peixes lisos (ou peixes de pele ou peixes de couro). Estas são categorias supra genéricas ou intermediárias (BERLIN, 1992), anteriormente descritas e conhecidas em comunidades ribeirinhas e outras cidades amazônicas (MURRIETA, 2001; BATISTA; PETRERE Jr., 2003; COSTA et al., 2013); e em comunidades de pescadores do litoral do Nordeste (COSTA NETO, 2000) e do Sudeste brasileiros (HANAZAKI; BEGOSSI, 2006). Estas categorias são mais ou menos congruentes entre as águas interiores da Amazônia e as regiões costeiras, mas na Amazônia os principais peixes lisos (Siluriformes) são os gêneros da Família Pimelodidae, enquanto que no litoral são os bagres da Família Ariidae.

Consumidores podem descrever outros fatores como determinantes na escolha do pescado para consumo, além do sabor. Ribeirinhos do alto rio Negro usaram os critérios densidade (quantidade de gordura), cheiro, cor, aparência (presença de sangue, dentes), comportamento e ecologia do peixe (dieta) na escolha das espécies preferidas (SILVA; BEGOSSI, 2007). Pescadores do litoral baiano levaram em consideração características comportamentais, morfológicas, toxicológicas, odoríferas e alimentares para classificar os peixes prescritos como alimentos (COSTA NETO, 2000). Enquanto ribeirinhos do baixo Amazonas descreveram o comportamento, a dieta, a cor, o cheiro e a aparência do peixe como importantes critérios no processo de escolha e classificação (MURRIETA, 2001). 
Devemos ressaltar que os peixes que chegam ao mercado local nem sempre são os mesmos que usualmente são utilizados e preferidos em comunidades ribeirinhas amazônicas. Um estudo sobre a pesca em área de manejo comunitário no Baixo Solimões revelou que os pescadores ribeirinhos dessa região usam um número maior de espécies para o consumo e selecionam um número menor (de espécies mais valorizadas) para atividades comerciais (BRAGA et al., 2008). No Baixo Amazonas esse comportamento foi verificado nas comunidades de várzea, nas pescarias feitas de acordo com espécies-alvo e ambiente escolhido, época do ano ou objetivo e tipo de pescaria (CERDEIRA; RUFFINO; ISAAC, 2000).

Com base nos tipos de pescarias realizadas e na forma de organização social, os pescadores do Baixo Solimões até o Baixo Amazonas foram classificados em duas categorias: pescador polivalente (ribeirinho ou lavrador) e pescador monovalente (ou comercial). Os primeiros dedicam-se a várias atividades durante o ano, habitam as áreas de várzea do rio Amazonas, pescam em família para o consumo próprio e sazonalmente para comercialização. Os segundos ocupam o seu tempo quase que exclusivamente nas atividades de pesca durante o ano todo para obter remuneração (FURTADO, 1990; BRAGA; BARROS; CHAVES, 2008; PEREIRA et al, 2007). Em Santarém, ambas as categorias de pescadores abastecem o mercado local, sendo que os pescadores polivalentes vendem a sua produção aos barcos especializados na compra de peixes nas áreas rurais (ISAAC; SILVA; RUFFINO, 2008).

A maioria dos comerciantes de Santarém (61\%) afirmou que vende essas espécies utilizadas para o consumo durante o ano todo, por serem as preferidas da população e estarem "disponíveis no mercado". Isso confirma a demanda do mercado local e a pressão que as mesmas espécies vêm sofrendo com o passar do tempo, o que é atestado pela sua presença constante em estudos de desembarque pesqueiro na Amazônia brasileira (PETRERE Jr., 1985; ISAAC; BARTHEM, 1995; BATISTA; PETRERE Jr., 2003; BATISTA et al., 2012; DORIA et al., 2012; ALCÂNTARA et al., 2015).

Os peixes preferidos antigamente, que os consumidores compravam e consumiam há pelo menos vinte anos eram o acari (Pterygoplichthys spp), a pescada (Plagioscion spp.) e os surubins (Pseudoplatystoma spp.) (Figura 4). É notável a mudança quanto às espécies preferenciais, confirmada pelo Teste-G, indicando que as preferências atuais pelas espécies de peixes não manteve as mesmas proporções de anos atrás (Teste-G = 66.6508; $(\mathrm{p})=<0.0001$; G.L.=17). Entre os motivos alegados pelos consumidores e confirmados pelos vendedores para essa mudança nas preferências, dois se destacaram e chamaram a atenção: (1) a indisponibilidade do produto no mercado; (2) a maior frequência de exemplares 
pequenos das espécies outrora preferenciais. Os vendedores confirmaram a preferência da população por exemplares grandes daquelas espécies, mas que quase já não os recebem para a revenda. $\mathrm{O}$ acari, em especial, foi um peixe cuja preferência por exemplares maiores e mais gordos foi notável. Comunitários do Baixo Amazonas revelaram preferência generalizada pela cabeça do acari, devido à concentração de carne e gordura ("tutano") na cabeça desse peixe (MURRIETA, 2001).

Figura 4 - Dez tipos de peixes citados como os preferenciais para o consumo antigamente em Santarém.

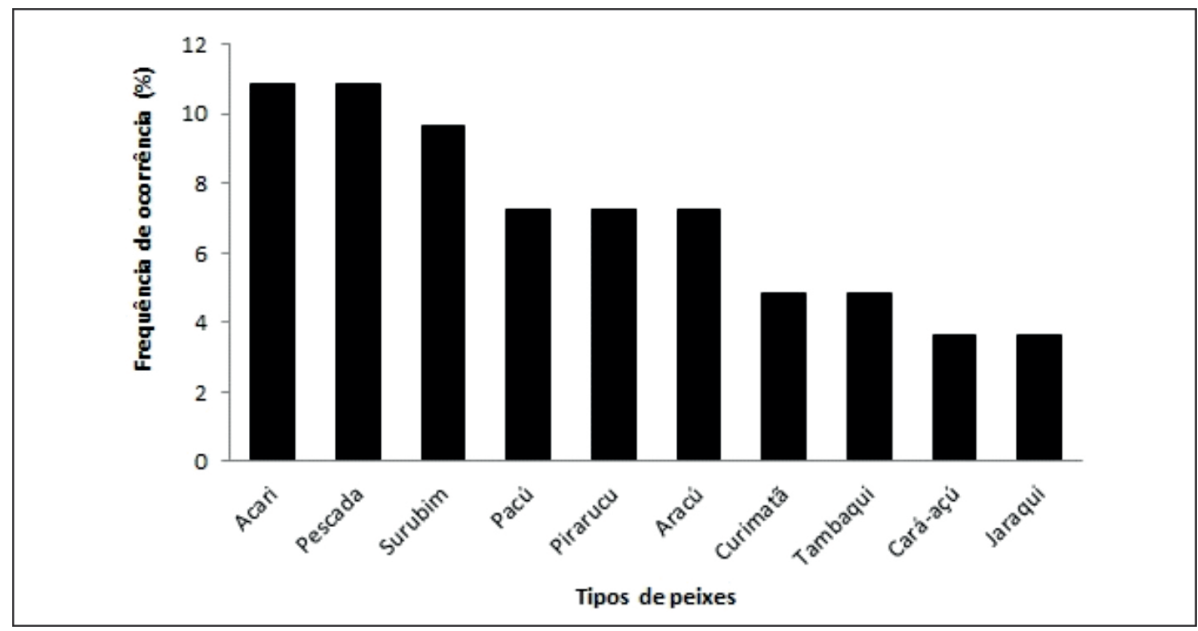

Fonte: Laboratório de Geoinformação e Análise Ambiental (ICTA/UFOPA).

Outro resultado notável foi que o tambaqui, peixe mais citado como preferencial para o consumo atualmente, não constava nem entre os cinco principais preferenciais para o consumo antigamente. A presença de tambaquis jovens desembarcados em Santarém foi percebida em estudos populacionais entre 1992 e 1993, que demonstraram que esta espécie estava em sobrepesca na região (ISAAC; RUFFINO, 1996). Há claros indícios da sobrepesca de tambaqui em vários pontos da Amazônia (FREITAS et al., 2007), com o agravante de que o mercado de exemplares imaturos é crescente, e sua presença nos locais de comercialização tornou-se uma rotina há mais de uma década. Em Santarém, nota-se neste estudo que essa pratica também é comum. Também é cada vez mais comum a presença de exemplares de tambaqui oriundos de cativeiro sendo vendidos nas feiras, principalmente vindos de outros estados, como Mato Grosso. Um tambaqui de lago da região chega a custar até $\mathrm{R} \$ 4,00$ a mais por quilo (kg), em comparação com o de cativeiro (U\$1,00 = R $\$ 2,26$ em julho de 2014). 
Parece cada vez mais necessário estimar os parâmetros populacionais de crescimento e mortalidade dessas espécies na região de Santarém, visando determinar qual a situação atual. Os acaris desembarcados em Santarém nos anos de 1992 e 1993 atingiram o tamanho máximo de $48 \mathrm{~cm}$, a pescada $68 \mathrm{~cm}$ e o surubim (tigre) $130 \mathrm{~cm}$ (RUFFINO; ISAAC, 1995), tamanhos que hoje os comerciantes afirmam não receber mais. Dados sobre o comprimento de captura e o esforço de pesca do surubim tigre entre 1993 a 1996 indicavam que o estoque dessa espécie no Médio Amazonas já estava sendo explorado em seu limite de sustentabilidade (RUFFINO; ISAAC, 1999).

Quando questionamos sobre a existência de algum tipo de peixe que não consumiam e o motivo (caso houvesse), a maioria dos consumidores entrevistados (52,6\%) afirmaram não consumir peixes lisos (Siluriformes) "de jeito nenhum". Entre os motivos alegados para não consumirem os peixes lisos estão principalmente: ser "reimoso, carregado, faz mal à saúde", "não gosto do sabor", "é feio, nojento" e o "preço alto". Mas, quase um quarto dos consumidores entrevistados $(24,5 \%)$ afirmou consumir todos os tipos de peixes sem nenhum problema. Os demais relataram que não consomem apenas os pequenos peixes de escamas (Characiformes e Perciformes) por apresentarem "muitas espinhas".

No litoral nordestino aquantidade de espinhas é um dos fatores que podem limitar o consumo de certos peixes, especialmente por crianças, (COSTA NETO, 2000). O baixo consumo de peixe liso em Parintins, no Amazonas, foi atribuído não apenas por serem considerados alimentos reimosos, mas também em virtude de fatores religiosos (COSTA et al., 2013). Os peixes lisos foram também os grupos mais citados pelos ribeirinhos no Alto rio Negro, como animais considerados reimosos, e que por isso devem ser evitados por pessoas que tenham feridas, erupções cutâneas, doenças inflamatórias ou ainda por mulheres nos períodos de menstruação, gravidez ou pós-parto (resguardo), que devem consumir somente os alimentos permitidos, como os peixes de escama, as aves domésticas (galinha e frango) ou pequenos animais que tenham a dieta à base de frutas (SILVA, 2007).

Certas espécies citadas como pouco consumidas atualmente, entre estas o acari, que tem "vindo muito pequeno"; e o pirarucu (Arapaima gigas), que embora seja uma espécie com excelente qualidade da carne e ter grande aceitação pela população para o consumo, foi citado como sendo pouco consumido devido ao preço elevado dos seus produtos (filé, peixe-seco, posta).

Não é recente a percepção pelas comunidades ribeirinhas da região da várzea de Santarém de que há tempos os acaris vêm sendo capturados muito jovens, e foi inclusive por solicitação dessas lideranças comunitárias que a 
captura e comercialização desta espécie está proibida desde 2004 nas regiões do Aritapera, Tapará e Ituqui (Instrução Normativa N ${ }^{\circ} 11,13$ e 19/2004) ; e em 2005 passou a ser proibida no período de defeso anual (01/12 a 30/03) em toda a Bacia Hidrográfica do rio Amazonas, no estado do Pará (Instrução Normativa $\mathrm{N}^{\circ}$ 22/2005).

Quando questionamos os comerciantes sobre a existência de mudanças com relação às espécies que chegavam aos mercados antigamente em comparação às espécies que chegam atualmente, a maioria (80\%) citou grupos que antes não eram explorados ou apresentavam baixas demandas e aceitação nos mercados e que hoje são vendidos, como é o caso de alguns peixes lisos: pirarara (Phractocephalus hemiliopterus), cujuba (Oxydoras niger) e bacus (Doradidae); das arraias (Potamotrygonidae) e peixes criados em cativeiro, com destaque para o tambaqui. Os motivos alegados pelos comerciantes para a comercialização destas espécies atualmente são vários, com destaque para: "aumentou a população e a procura", "são bons pra filé e pra exportação", "falta outros peixes no mercado", "é gostoso, quem experimenta gosta e passa a consumir" e "o preço baixo". Porém, cerca de $20 \%$ dos entrevistados afirmaram que sempre venderam os mesmos peixes.

A pesca de Siluriformes na Amazônia teve grande expansão nos últimos 40 anos, e está ligada à instalação de frigoríficos qualificados para a preparação e principalmente exportação da produção pesqueira (BARTHEM; GOULDING, 1997; BATISTA; PETRERE, 2003; SANTOS; SANTOS, 2005). Aparentemente, hoje há limitações econômicas, não ecológicas ou determinadas por órgãos ambientais atuando na pesca de peixes lisos, e que de alguma forma influenciam o comportamento do pescador a buscar um pescado com tamanho que forneça um rendimento mínimo de filé, que dê condições de repassar o custo do trabalho e das despesas associadas para os frigoríficos (BATISTA; FREITAS, 2003).

Apesar da persistência do tabu contra o consumo de Siluriformes entre os consumidores, é notório que a rejeição ao consumo está diminuindo na região e, com isso, os preços estão aumentando, conforme informado pelos consumidores. A existência ou não de exploração/uso/consumo pode ser em função da demanda de mercado e da disponibilidade das espécies-alvo, enquanto que a quebra de um tabu na sociedade representaria uma mudança cultural (aspecto antropológico), assim como a perda da "proteção" que essas etnoespécies recebem poderia resultar em um problema biológico (a sobrepesca) (BATISTA; PETRERE, 2003). 


\section{CONSIDERAÇÕES FINAIS}

Entre os dez tipos de peixes (etnoespécies) citados como preferidos para consumo atualmente, nove são os mesmos peixes consumidos antigamente, mas houve grandes mudanças na preferência. O consumo se concentra na Amazônia, entre 6 e 12 espécies que respondem por $80 \%$ do que é desembarcado nos principais portos (BARTHEM; FABRÉ, 2004), mas a oferta local é diversificada (Tabela 1). O tambaqui que é de longe o preferido atualmente, há anos vem dando sinais de sobrepesca (PETRERE Jr, 1983; ISAAC; RUFFINO, 1996; FREITAS et al., 2007), mas subiu bastante no ranking da preferência. A pescada e o acari, que já foram os mais preferidos tiveram as maiores quedas no ranking, enquanto o acará-açu saiu do grupo dos dez mais consumidos, o tucunaré surge como a segunda espécie em preferência. Aliás, as duas espécies mais preferidas atualmente (tambaqui e tucunaré) podem incluir populações significativas de cultivo. Aparentemente o acari perdeu a preferência devido à população não gostar de consumir indivíduos jovens, ao contrário dos tambaquis pequenos e imaturos, que são bem aceitos e denominados de "ruelo".

As espécies de peixes ameaçadas geralmente são muito apreciadas para o consumo, preferencialmente de tamanho grande; quando exploradas intensamente, as espécies com baixa taxa de crescimento devem repor lentamente o seu estoque de adultos. Esta mudança vai disparar o primeiro sinal do efeito da pesca: a diminuição do número de exemplares maiores e a captura de indivíduos cada vez mais jovens (ISAAC; BARTHEM 1995).

Preferências e aversões alimentares resultam da interação entre diversos fatores - necessidades e restrições individuais, condições socioeconômicas, sazonalidade (ciclos ecológicos dos recursos naturais), e da dinâmica sóciopolítico-econômica dos mercados (MURRIETA, 2001). Os tabus são regras locais que afetam as necessidades e preferências, mas estas nem sempre são obedecidas, pois o alimento proibido pode vir a ser a única opção para o consumidor que é, entretanto, consciente dos riscos associados à quebra do tabu, dependendo do contexto e do momento (SILVA, 2007). No mercado de Santarém, tabus e preços influenciam as escolhas dos consumidores de peixes.

\section{AGRADECIMENTOS}

Agradecemos à FAPESPA pela bolsa concedida à A. A. Silva. 


\section{REFERÊNCIAS}

ALCÂNTARA, N. C.; GONÇALVES, G. S.; BRAGA, T. M. P.; SANTOS, S. M.; ARAÚJO, R. L.; PANTOJA-LIMA, J.; ARIDE, P. H. R.; OLIVEIRA, A.T. Avaliação do desembarque pesqueiro (2009-2010) no município de Juruá, Amazonas, Brasil. Biota Amazônia, Macapá, v. 5, n. 1, p. 37-42, 2015.

BATISTA, V. S.; INHAMUNS, A. J.; FREITAS, C. E. C.; FREIRE-BRASIL, D. Characterization of the fishery in river communities in thelow-Solimões / highAmazon region. Fisheries Management and Ecology, v. 5, p. 419-435, 1998.

BATISTA, V. S.; PETRERE JR., M. Characterization of the commercial fish production landed at Manaus, Amazonas State, Brazil. Acta Amazonica, v. 33, n. 1, p. 53-66, 2003.

BATISTA, V. S.; FREITAS, V. S. O descarte de pescado na pesca com rede de cerco no Baixo Rio Solimões, Amazônia Central. Acta Amazonica, v. 33, n. 1, p. 123-143, 2003.

BATISTA, V. S.; ISAAC V. J; VIANA, J. P. A pesca na Amazônia Central. In: RUFFINO, M. L. A pesca e os recursos pesqueiros na Amazônia brasileira. Manaus: Ibama/ProVárzea, 2004. p. 213-244.

BATISTA, V. S.; ISAAC, V. J.; FABRÉ, N. N. Produção desembarcada por espécie e sua variação por macrorregião amazônica. In: BATISTA, V. S. ISAAC, V. J. Peixes e pesca no Solimões-Amazônia: uma avaliação integrada. Brasília: Ibama/Pro Várzea, 2012. p. 108-133.

BARTHEM, R. B.; GUERRA, H.; VALDERRAMA, M. Diagnóstico de los Recursos Hidrobiologicos de la Amazonia. [s.l.]: Tratado de Cooperación Amazonica. Secretaria Pro Tempore, 1995. 162 p.

BARTHEM, R.; GOULDING, M. Os bagres balisadores: ecologia, migração e conservação de peixes amazônicos. Tefé: Sociedade Civil Mamirauá; Brasília: CNPq, 1997. 140p.

BARTHEM, R. B.; FABRÉ, N. N. Biologia e diversidade dos recursos pesqueiros da Amazônia. In: RUFFINO, M. L. A pesca e os recursos pesqueiros na Amazônia brasileira. Manaus: Ibama/ProVárzea, 2004. p.17-62.

BAYLEY, P. B. Fish yield from the Amazon in Brazil: comparisons with African river yields and management possibilities. Transactions of the American Fisheries Society, v. 110, p. 351-359, 1981. 
BRAGA, T. M. P.; BARROS, J. F.; CHAVES, M. P. S. R. Pesca e conflitos socioambientais na Amazônia Central: estudo em uma área com manejo comunitário. Somanlu, Manaus, v. 7, p. 107-119, 2008.

BRAGA, T. M. P.; REBÊLO, G. H. Conhecimento tradicional dos pescadores do baixo rio Juruá: aspectos relacionados aos hábitos alimentares dos peixes da região. Interciência, p. 659-665, 2014.

BEGOSSI, A., PETRERE Jr., M. Utilização de recursos aquáticos e tecnologia entre pescadores do Médio Tocantins (GO e MA). In: ENCONTRO DE CIÊNCIAS SOCIAIS E O MAR NO BRASIL, 2., 1988. São Paulo. Anais... São Paulo: IOUSP/F.FORD/UICN, 1988. p. 158-169.

BERLIN, B. Ethnobiological classification: Principles of categorization of plants and animals in traditional societies. Princeton; New Jersey: Princeton University Press, 1992. 335p.

BITTENCOURT, M.; COX-FERNANDES, C. Pesca Comercial na Amazônia Central: uma atividade sustentada por peixes migradores. Ciência Hoje, v.11, n. 64, p. 20-24, 1990.

CERDEIRA, R. G. P.; RUFFINO, M. L.; ISAAC, V. J. Fish catches among riverside communities around Lago Grande de Monte Alegre, Lower Amazon, Brazil. Fisheries Management and Ecology, v. 7, p. 355-374, 2000.

COSTA, T. V.; SILVA, R. R. S.; SOUZA, J. L.; BATALHA, O. S.; HOSHIBA, M. A. Aspectos do consumo e comércio de pescado em Parintins. Bol. Inst. Pesca, São Paulo, v. 39, n. 1, p. 63-75, 2013.

COSTA-NETO, E. M. Restrições e Preferências Alimentares em Comunidades de Pescadores do Município de Conde, Estado da Bahia, Brasil. Rev. Nutr., Campinas, v. 13, n. 2, p. 117-126, maio/ago. 2000.

DORIA, C. R. D. C.; RUFFINO, M. L.; HIJAZI, N. C.; CRUZ, R. L. D. A pesca comercial na bacia do rio Madeira no estado de Rondônia, Amazônia brasileira. Acta Amazonica, v. 42, n. 1, p. 29-40, 2012.

FABRÉ, N. N.; ALONSO, J. C. Recursos ícticos no Alto Amazonas: sua importância para as populações ribeirinhas. Bol. Mus. Para. Emílio Goeldi, ser. Zool. v. 14, n. 1, p. 19-55, 1998.

FREITAS, C. E. C.; NASCIMENTO, F. A.; SOUZA, F. K. S. Levantamento do estado de explotação dos estoques de curimatã, jaraqui, surubim e tambaqui. In: O setor pesqueiro na Amazônia: análise da situação atual e tendências do desenvolvimento a indústria da pesca/Projeto Manejo dos Recursos Naturais da Várzea. Manaus: Ibama/ProVárzea, 2007. p. 77-100. 
FURTADO, L. G. Características gerais e problemas da pesca amazônica no Pará. Bol. Mus. Para. Emílio Goeldi, sér. Antropol. v. 6, n. 1, 1990.

GOULDING, M. Ecologia da pesca no rio Madeira. Manaus: CNPq/INPA, 1979. 172p.

GONZÁLES, C. G.; FELPETO, A. B.; ESTRAVIZ, I. M.; ALARCÓN, I. R.; CASTAÑO, A. R. V.; LISTE, A. V. Tratamiento de datos. Vigo: Universidad de Vigo, Edicione Diaz de Santos, 2006. 357p.

HANAZAKI, N; BEGOSSI, A. Catfish and mullets: the food preferences and taboos of Caiçaras (Southern Atlantic Forest Coast, Brazil). Interciência, v. 31, n. 2, p. 123-129, 2006.

IBGE.. Censo Demográfico 2010. Disponível em: <http://www.cidades.ibge. gov.br $/$ painel $/$ populacao.php?lang $=\& \operatorname{codmun}=150680 \&$ search $=\mid$ santarem $>$. Acesso em: 20 jan. 2016.

ISAAC, V. J.; BARTHEM, R. B. Os recursos pesqueiros da Amazônia brasileira. Bol. Mus. Para. Emílio Goeldi, sér. Antropol., Belém, v. 11, n. 2, p. 295-339, 1995.

ISAAC, V. J.; RUFFINO, M. L. Population dynamics of tambaqui, Colossoma macropomum Cuvier, in the lower Amazon, Brazil. Fisheries Management and Ecology, v. 3, p. 315-333, 1996.

ISAAC, V. J.; SILVA, C. O.; RUFFINO, M. L. The artisanal fishery fleet of the lower Amazon. Fisheries Management and Ecology, v. 15, n. 179-187, 2008.

MARQUES, J. G. W. Aspectos Ecológicos na Etnoictiologia dos pescadores do Complexo Estuarino-Lagunar Mundaú-Manguaba, Alagoas. 1991. 280f. Tese (Doutorado) - Universidade Estadual de Campinas. Campinas, 1991.

MACBETH, H.; LAWRY, S. Food Preferences and Taste. In: MACBETH, H. Food Preferences and Taste. Oxford: Berghahn Books. 1997, p. 5-13.

MURRIETA, R. S. S. Dialética do sabor: alimentação, ecologia e vida cotidiana em comunidades ribeirinhas da Ilha de Ituqui, Baixo Amazonas, Pará. Revista de Antropologia USP, v. 44, n. 2, p. 39-88, 2001.

PETRERE JR., M. 1983. Yield per recruit of the tambaqui, Colossoma macropomum Cuvier, in the Amazonas State, Brazil. Journal of Fish Biology, v. 22, p. 133144, 1983.

PETRERE JR., M. 1985. A pesca comercial no rio Solimões-Amazonas e seus afluentes: Análise dos informes do pescado desembarcado no Mercado Municipal de Manaus (1976-1978). Ciência e Cultura, v. 37, 1987-1999. 
PEREIRA, H. S.; SOUZA, D. S. R.; RAMOS, M. M. A diversidade da pesca nas comunidades da área focal do projeto PIATAM. In: FRAXE, T. J. P.; PEREIRA, H. S.; WITKOSKI, A. C. (Orgs.). Comunidades ribeirinhas amazônicas: modos de vida e uso dos recursos naturais. Manaus: EDUA, 2007. p. 171-195.

RIBEIRO, M. C. L. B.; PETRERE JR., M. Fisheries ecology and management of the jaraqui (Semaprochilodus taeniurus, S. insignis) in Central Amazônia. Regulated Rivers: Research and Management, v. 5, p. 195-215, 1990.

RUFFINO, M. L.; ISAAC, V. J. The fisheries of the Lower Amazon: questions of management and development. Acta Biologica Venezuelica, v. 15, n. 2, p. 37-46, 1994.

RUFFINO, M. L.; ISAAC, V. J. Life cycle and biological parameters of several Brazilian Amazon fish species. Naga ICLARM Q, v. 18, n. 4, p. 41-45, 1995.

RUFFINO, M. L.; BARTHEM, R. B. Perspectivas para el Manejo de los Bagres Migradores de la Amazonia. Boletín Científico, Santa Fé de Bogotá, n. 4, p.1928, 1996.

RUFFINO, M. L.; ISAAC, V. J. Dinâmica populacional do surubim-tigre, Pseudoplatystoma tigrinum (Valenciennes, 1840) no Médio Amazonas (Siluriformes, Pimelodidae). Acta Amazonica, v. 29, p. 463-476. 1999.

SANTOS, G. M.; SANTOS, A. C. M. Sustentabilidade da pesca na Amazônia. Estudos Avançados, 19(54): p. 165-182, 2005.

SILVA, A. L. Comida de gente: preferências e tabus alimentares entre os ribeirinhos do Médio Rio Negro (Amazonas, Brasil). Departamento de Ecologia - Unesp. Revista de Antropologia, São Paulo, v. 50. n. 1, p. 125-179, 2007.

SILVA, A. L.; BEGOSSI, A. Biodiversity, Food Consumption and ecological niche dimension: a study case of the riverine populations from the Rio Negro, Amazonia, Brazil. Environment, Development and Sustainability, v. 11, n. 3, p. 1-24, 2007.

SILVANO, R. A. M.; BEGOSSI, A. Ethnoichthyology and fish conservation in the Piracicaba river (Brasil). Journal of Ethnobiology, v.22. n.2, p. 285-306., 2001.

SMITH, N. H. A pesca no Rio Amazonas. Manaus: INPA/CNPq, 1979. 154 p. 